\title{
THE ROLE OF INTRAOPERATIVE FROZEN SECTION OF SENTINEL LYMPH NODES IN UPFRONT BREAST CONSERVATION CANCER SURGERY
}

\author{
Ruqayya N. Khan ${ }^{1}$, Awais A. Malik ${ }^{1}$ Sameen Mohtasham¹, Amina I. Khan, \\ Muhammad A. Parvaiz ${ }^{1}$, Asif Loya ${ }^{2}$ \\ ${ }^{1}$ Department of Surgery, Shaukat Khanum Memorial Cancer Hospital and Research Centre, Lahore, Pakistan, \\ ${ }^{2}$ Department of Pathology, Shaukat Khanum Memorial Cancer Hospital and Research Centre, Lahore, Pakistan \\ Received: 08 March 2019 / Accepted: 10 June 2019
}

\begin{abstract}
Objective: Sentinel lymph node biopsy is the standard of care in clinically negative axilla in breast cancer patients for which frozen section (FS) is routinely performed intraoperatively. The objective of this study was to justify the use of FS in terms of number of tests performed and their impact on decision-making and cost saving.

Materials and Methods: We retrospectively reviewed our prospectively maintained data from January 2014 to January 2018 for intraoperative FS in upfront breast conservation surgery patients.

Results: A total of 357 patients were studied. All were female. Median age was 50 years (24-84). Mean tumour size was $29.11 \mathrm{~mm}$. Numbers of sentinel lymph nodes identified were 1 in 50 (14.2\%) patients, 2 in 121 (33.89\%) patients and $\geq 3$ in $186(52 \%)$ patients. Number of positive sentinel lymph nodes was 0 in 264 (73.9\%) patients, 1 in 62 (17.4\%) patients, 2 in $20(5.6 \%)$ patients and $\geq 3$ in $11(3.08 \%)$ patients. Axillary lymph node dissection (ALND) was offered to 30 (8.4\%) patients as per the American College of Surgeons Oncology Group Z0011. The results for ALND showed that only $8(2.3 \%)$ out of 30 patients had positive nodes identified in the additional axillary nodes dissected. Sensitivity of FS was $97 \%$ and specificity was $98.86 \%$. False-negative rate was $3.22 \%$.
\end{abstract}

Conclusion: Intraoperative FS can be safely omitted in early breast cancer patients undergoing upfront breast conservation cancer surgery due to high sensitivity and specificity leading to low false-negative rates. ALND can be performed as a second operation as warranted only in a minority of patients.

Key words: American College of Surgeons Oncology Group Z0011 trial, axillary lymph node dissection, intraoperative frozen section, sentinel lymph node biopsy

\section{Introduction}

Lymph node status is one of the most important predictors of survival and recurrence in breast cancer. Axillary lymph node dissection (ALND) has been a major part of breast cancer treatment since the very beginning when William S Halsted, a young surgeon at John Hopkins, first introduced radical mastectomy in $1882 .{ }^{[1]}$ It involved the removal of entire breast, surrounding tissues, pectoral muscles and axillary lymph nodes. He hypothesised that breast cancer spreads in an orderly fashion and spreads locally first followed by axillary lymph nodes and then to

Correspondence: Ruqayya N. Khan, Department of Surgery, Shaukat Khanum Memorial Cancer Hospital and Research Centre, Lahore, Pakistan. Email: rocksj_84@hotmail.com other organs of the body. Later came the era of modified radical mastectomy in 1970s when results of NSABP-04 trial results were published. It supported the paradigm shift to less radical surgery for breast cancer. ${ }^{[2]}$ To further minimise the extent of surgery NSABP-06, a randomised controlled trial comparing lumpectomy and ALND with or without breast irradiation with total mastectomy and ALND (modified radical mastectomy) in patients with tumours $4 \mathrm{~cm}$ or less was conducted. ${ }^{[3]}$ The NSABP B-06 trial established the concept of breast-conserving surgery and confirmed the importance of radiation as a component of such treatment.

ALND is associated with high morbidity due to complications such as lymphoedema, pain, paraesthesia 
and shoulder movement restriction. Sentinel lymph node biopsy (SLNbx) was first reported in 1994. ${ }^{[4]}$ Compared to ALND, SLNbx is associated with less morbidity and complications such as impaired shoulder function, paraesthesia and lymphoedema. ${ }^{[5,6]}$

In early 2000 NSABP B-32, a randomised controlled trial enrolled 5611 patients and randomised them to undergo SLNbx and ALND (Group 1) or SLNbx with ALND only if the sentinel lymph node was positive. It concluded that sentinel lymph node dissection is enough for patients whose sentinel nodes are negative and that there is no need to proceed to complete axillary dissection. ${ }^{[7]}$ In 1999, the American College of Surgeons Oncology Group (ACOSOG) launched the Z0011 trial that randomised early stage (T1-2, clinically N0) breast cancer patients with a tumour-positive sentinel node to completion ALND versus observation. ${ }^{[8]}$ It was concluded that patients having 1-2 positive lymph nodes followed by radiotherapy can be omitted from undergoing ALND. However, patients with 3 or more positive nodes need to undergo complete axillary dissection.

In early breast cancer, an intraoperative frozen section (FS) of sentinel lymph nodes is performed routinely to assess their status and perform ALND if metastasis found avoiding the second operation. The sensitivity of FS ranges from $58 \%$ to $76 \%$ depending on tumour characteristics and method of pathological examination. ${ }^{[9-12]}$ It provides result within an average time of 20-30 $\mathrm{min}$. However, its use has become controversial in early breast cancer due to extra cost, increased time and possible false-negative rates. In addition, landmark trials like ACOSOG Z0011 ${ }^{[13]}$ have further questioned the benefit of ALND in cases of 1 or 2 positive sentinel lymph nodes, making management decisions more controversial which should be more appropriately made in a multidisciplinary meeting rather than on intraoperative assessment.

The aim of this study was to justify the use of intraoperative FS in upfront breast conservation cancer surgery in this era of controversy with regard to false-negative rates, sensitivity and specificity to assess its accuracy in our hospital and also to justify the use of FS in terms of number of tests performed and their impact on decision-making and cost saving.

\section{Materials and Methods}

An institutional prospectively maintained database was utilised at Shaukat Khanum Memorial Cancer Hospital and Research Centre, Pakistan, to retrospectively review patients from January 2014 to January 2018 after taking Institutional Review Board approval. All the patients with invasive tumour requiring breast conservation surgery and SLNbx for T1-T2 tumours with clinically negative axilla which includes patients with no suspicious nodes on clinical examination as well as on radiological investigations were included in the study. Patients with ductal carcinoma in situ were excluded from the study. Prior neoadjuvant chemotherapy patients were also excluded, so only upfront surgery patients were reviewed. ACOSOG Z0011 trial criterion ${ }^{[10]}$ was applied on all patients as our standard hospital guidelines. As per the criteria, only patients with tumours $<5 \mathrm{~cm}$ and no clinically palpable lymph nodes underwent upfront surgery with a SLNbx. Patients with single positive node were spared of ALND while ALND was performed in 3 or more positive lymph nodes according to the Z-11 criteria. ${ }^{[10]}$ However, in patients with 2 positive lymph nodes, ALND was omitted if tumour was of good tumour biology, i.e., oestrogen receptor/progesterone receptor (ER/PR) positive and Her2Neu negative.

Sentinel lymph nodes were identified by injecting Tc-99m human serum albumin (HSA) a day before surgery. A dose of $40 \mathrm{MBq}$ was injected at periareolar location. Lymphoscintigraphy was performed at $30 \mathrm{~min}$ after injection. In case of non-visualization of sentinel lymph node on planar scintigraphy, further delayed planar and/or SPECT/CT (single photon emission comupted tomography/computed tomography) images were performed. A gamma probe was used for sentinel lymph node localisation. Blue dye (blue patente V Guerbet 2.5\%) was used in addition to Tc-99m HSA in some patients at the time of surgery where there was no localisation of sentinel lymph nodes by Tc-99m HSA. $2 \mathrm{ml}$ of blue dye was injected subcutaneously at periareolar location. Lymph nodes which were suspicious on palpation were also sent for FS.

Sentinel lymph nodes were sent for FS in all patients immediately. After lymph nodes were received in pathology department, they were cut according to the standard sentinel protocol. Tissue was then placed on a metal disc which was frozen rapidly to about $-20-30^{\circ} \mathrm{C}$ by embedding in 
Table 1: Patients characteristics

\begin{tabular}{|c|c|}
\hline Characteristics & Data (n 357) \\
\hline Age (years): Median (range) & $50(24-84)$ \\
\hline Dominant side (Left) & 197 \\
\hline Median BMI (range) & $30(16-59)$ \\
\hline Median tumour size (mm) & 25 \\
\hline \multicolumn{2}{|l|}{ Nodal status } \\
\hline NO & 264 \\
\hline N1 & 93 \\
\hline \multicolumn{2}{|l|}{ Histopathological type } \\
\hline Invasive ductal Ca & 344 \\
\hline Invasive lobular $\mathrm{Ca}$ & 13 \\
\hline Median operative time ( $\mathrm{min}$ ) & 70 \\
\hline \multicolumn{2}{|l|}{ Receptor status } \\
\hline ER positive & 257 \\
\hline PR positive & 214 \\
\hline Her2Neu positive & 56 \\
\hline \multicolumn{2}{|l|}{ Histopathology grade } \\
\hline Grade I & 10 \\
\hline Grade II & 175 \\
\hline Grade III & 172 \\
\hline \multicolumn{2}{|c|}{ Number of SLNs identified at SLNbx } \\
\hline 1 & 50 \\
\hline 2 & 121 \\
\hline 3 & 145 \\
\hline 4 & 34 \\
\hline 5 & 4 \\
\hline 6 & 1 \\
\hline 7 & 1 \\
\hline 8 & 1 \\
\hline \multicolumn{2}{|l|}{ Size of metastatic deposit } \\
\hline Macromet & 66 \\
\hline Micromet & 23 \\
\hline
\end{tabular}

BMI: Body mass index, SLNbx: Sentinel lymph node biopsy, SLNs: Sentinel lymph node, ER: Oestrogen receptor, PR: Progesterone receptor

optimal temperature cutting compound. The tissue was then placed in a cryostat, where $1 \mathrm{~mm}$ sections were taken using a microtome. The sections were placed on glass slides and stained with haematoxylin and eosin. The slides were then reviewed by two experienced pathologists who then inform the referring surgeon about the diagnosis. FS and definitive section results were compared regarding the pathological diagnosis for the SLNs.
Patient age, body mass index (BMI), side of tumour, tumour size, histological tumour type, grade, ER/PR and HER2Neu status, duration of surgery and hospital stay were also recorded. Size of lymph node metastasis if any was also recorded both intraoperatively as reported by pathologist on FS and on final histopathology report and the results were compared. $<2 \mathrm{~mm}$ was taken as micrometastases, more than $2 \mathrm{~mm}$ as macrometastases and isolated tumour cells as single tumour cells or cluster of cells less than $0.2 \mathrm{~mm}$.

Data were entered in SPSS version 20 and analysed through its statistical programme. False-negative cases were defined as patients in whom FS at the time of surgery was reported as negative but was found to be positive on final pathology.

\section{Results}

A total of 357 patients were studied. All were female. Median age was 50 years (24-84). Median BMI was $30 \mathrm{~kg} / \mathrm{m}^{2}$ (16-59). 197 (55.18\%) patients had left-sided tumour while 160 (44.81\%) had right-sided tumour. Average tumour size was $29.11 \mathrm{~mm}$. Predominant histological type was invasive ductal carcinoma in $344(96.35 \%)$ patients while $13(3.65 \%)$ patients had invasive lobular carcinoma. 172 (48.17\%) patients had Grade III tumour, 175 (49\%) had Grade II, whereas 10 (2.8\%) patients had Grade I tumour. 257 (72\%) patients were ER positive, 214 (59.9\%) were PR positive and only $56(15.7 \%)$ patients were Her2Neu positive. Mean operative time was 70 min while mean hospital stay was 1 day (0-4) [Table 1].

Median numbers of sentinel lymph nodes identified were 1 in $50(14.2 \%)$ patients, 2 in $121(33.89 \%)$ patients and $\geq 3$ in $186(52 \%)$ patients. All patients had sentinel node identified through either blue dye or Tc-99m. Median number of positive sentinel lymph nodes was 0 in $264(73.9 \%)$ patients, 1 in $62(17.4 \%)$ patients, 2 in $20(5.6 \%)$ patients and $\geq 3$ in $11(3.08 \%)$ patients. ALND was offered to $30(8.4 \%)$ patients [Table 2]. All the patients with 3 or more positive lymph nodes $11 / 11(100 \%)$ were offered ALND following ACOSOG Z0011 criteria. 7 patients had 2 lymph nodes positive and 12 patients had only 1 lymph node positive, but ALND was performed due to larger metastatic deposit, visible metastatic disease or larger breast tumour size with poor tumour biology. The results for ALND showed that only 8 (2.3\%) out of 30 patients who had positive nodes identified in the 
Table 2: Number of positive (Macromets) LNs identified at SLNBx

\begin{tabular}{l|c|c|c}
\hline Number of lymph nodes & Frequency & Percentage & Cumulative percent \\
\hline 0 & 264 & 73.9 & 73.9 \\
\hline 1 & 62 & 17.4 & 91.3 \\
\hline 2 & 20 & 5.6 & 96.9 \\
\hline 3 & 10 & 2.8 & 99.7 \\
\hline 6 & 1 & 0.3 & 100 \\
\hline Total & 357 & & \\
\hline
\end{tabular}

SLNBx: Sentinel lymph node biopsy, LN: Lymph node

Table 3: Comparison of frozen section and final histopathology

\begin{tabular}{l|l|l|c|c}
\hline Parameters & Frozen section & Final histopathology & Frequency & Percentage \\
\hline TP & Positive & Positive & 90 & 25.21 \\
\hline TN & Negative & Negative & 261 & 73.10 \\
\hline FN & Negative & Positive & 3 & 0.80 \\
\hline FP & Positive & Negative & 3 & 0.80 \\
\hline
\end{tabular}

TP: True positive, TN: True negative, FN: False negative, FP: False positive

Table 4: Intraoperative frozen section report according to luminal type

\begin{tabular}{l|c|c|c|c|c}
\hline Molecular sutype & Negative & $\mathbf{1} \mathbf{L N}^{\text {+ve }}$ & $\mathbf{2} \mathbf{L N s}^{\text {+ve }}$ & 3 or $\mathbf{~ m o r e ~} \mathbf{L N s}^{\text {+ve }}$ & Total \\
\hline Luminal A & 125 & 39 & 15 & 10 & 189 \\
\hline Luminal B & 57 & 7 & 3 & 1 & 68 \\
\hline TNBC & 65 & 14 & 1 & 0 & 80 \\
\hline Her2 Enriched & 16 & 2 & 1 & 0 & 19 \\
\hline Total & 263 & 62 & 20 & 11 & 356 \\
\hline
\end{tabular}

additional axillary nodes dissected. So out of a total of 357 patients, only $30(8.4 \%)$ patients needed an ALND out of which only $8(2.3 \%)$ patients had disease beyond the identified sentinel node which included most of those patients who had 3 positive lymph nodes on FS. Hospital stay was increased by an average of 1 day in case of ALND.

FS reports of sentinel lymph nodes were compared to final histopathology [Table 3]. True-positive nodes were found in $90(25.21 \%)$ patients and true negatives were identified in $261(73.1 \%)$ patients. Only $3(0.8 \%)$ patients had false-negative reports along with $3(0.8 \%)$ other having false-positive reports. None of the falsenegative patients required ALND as only 1 lymph node was positive, and ALND would have been avoided even if they were positive on intraoperative FS. Likewise, false- positive patients also had only 1 lymph node positive on intraoperative FS so ALND was avoided.

FS reports were also evaluated according to molecular subtypes to assess which tumour biology is more likely to have positive lymph nodes. 39 patients with single positive lymph nodes, 15 with 2 positive lymph nodes and 10 with 3 or more positive lymph nodes were of luminal A type $\left(\mathrm{ER}^{+\mathrm{ve}}, \mathrm{PR}^{+\mathrm{ve}}\right.$ and $\left.\mathrm{Her} 2 \mathrm{Neu}^{-\mathrm{ve}}\right) .7$ patients with single positive lymph nodes, 3 with 2 positive lymph nodes and 1 with 3 or more positive lymph nodes were of luminal B type $\left(\mathrm{ER}^{+\mathrm{ve}}, \mathrm{PR}^{-\mathrm{ve}}\right.$ and $\left.\mathrm{Her} 2 \mathrm{Neu}^{+\mathrm{ve}}\right) .14$ patients with single positive lymph nodes, 1 with 2 positive lymph nodes and none with 3 or more positive lymph nodes were of triple-negative breast cancer (TNBC) type (ER ${ }^{-v e}, \mathrm{PR}^{-\mathrm{ve}}$ and $\mathrm{Her} 2 \mathrm{Neu}^{-\mathrm{ve}}$ ). 2 patients with single positive lymph nodes, 1 with 2 positive lymph nodes and none with 3 or 
Table 5: Sensitivity and specificity of frozen section

\begin{tabular}{l|l|c}
\hline Parameter & Calculations & Value (\%) \\
\hline Sensitivity & TP/TP+FN & 97 \\
\hline Specificity & TN/TN+FP & 98.86 \\
\hline False-negative rate & FN/TP+FN & 3.22 \\
\hline PPV & TP/TP+FP & 96.77 \\
\hline NPV & TN/TN+FN & 98.86 \\
\hline Accuracy & TP+TN/TP+TN+FP+FN & 98.31 \\
\hline
\end{tabular}

PPV: Positive predictive value, NPV: Negative predictive value, TP: True positive, TN: True negative, FN: False negative, FP: False positive

more positive lymph nodes were Her2 enriched (ER ${ }^{-v e}$, $\mathrm{PR}^{-\mathrm{ve}}$ and $\mathrm{Her} 2 \mathrm{Neu}^{+\mathrm{ve}}$ ) [Table 4].

Sensitivity of FS in our hospital was $97 \%$ while specificity of $98.86 \%$. False-negative rates were calculated to be $3.22 \%$. Positive predictive value was $96.77 \%$ and negative predictive value was $98.86 \%$ [Table 5].

\section{Discussion}

SLNbx is the current standard for all patients with early breast cancer undergoing upfront surgery. All nodes retrieved are sent for FS and those patients with 3 or more positive nodes undergo axillary dissection. Our study has shown that the number of patients requiring an ALND after SLNBx is very low. Only 30 out of 357 patients needed an axillary dissection. For identifying one patient who merits an axillary dissection, a total of $12 \mathrm{FS}$ s were performed.

It is important to discuss that 264 patients had a negative axilla and required no ALND. 73 patients who had a positive SLNBx did not require ALND. That means that for 327 patients the SLNB had no role in decision-making regarding axillary dissection. Keeping this in mind, we can say that a FS has a limited role in decision-making after SLNBx in early breast cancer. For this reason, a lot of studies have questioned the role of a routine FS for SLNBx in early breast cancer. ${ }^{[14,15]}$

Rónká et al. in a prospective trial showed that, with respect to hospital costs, FS analysis seems to be worthwhile as long as the false-negative rate does not exceed $35 \% \cdot{ }^{[16]}$ The false-negative rate in our study was $3.22 \%$ which meets international standards. The reported rates are between $2.8 \%$ and $4.9 \%$ [Table 3$]^{[17,18]}$

Julie Anne Bishop in her study showed that since the Z0011, intraoperative sentinel lymph node evaluation has significantly decreased as only $3 \%$ of patients underwent ALND and 97\% (147 of 151) of the patients were spared ALND. Therefore, routine FS diagnosis for sentinel lymph node biopsies can be avoided in these patients. ${ }^{[14]}$

Leonardo Russo in a retrospective analysis of 281 patients showed that comprehensive information of the definitive histopathological findings either of the primary tumour and SLNs may help in making a better decision to completion ALND without an intraoperative FS analysis. The false-negative rate in SLN with macrometastasis was $13.1 \%$ and for micrometastasis cases was $66.7 \%$ $(P<0.001){ }^{[19]}$

Another aspect of omitting a FS is cost saving. Our current practice is to perform FS intraoperatively in all early breast cancer patients with clinically negative axilla to confirm axillary metastasis and avoid unnecessary ALND.

The cost of one FS in Pakistan is 95 USD and it requires minimum of 20-30 min for processing by pathology department. As reported in our study out of 357 patients only 30 patients required ALND. For identifying one patient who merits an axillary dissection, a total of 12 FSs were offered which increases the cost to 1140 USD. If we omit this FS, we can save considerable cost and time. Cost is increased further in the form of manpower as substantial amount of work is required by laboratory technicians and pathologists. In addition, longer operation room time utilisation as the length of procedure may be increased further on the result of FS and sometimes due to increased waiting time leading to delay of surgeries and even cancellations. However, doing a second operation can have emotional impact on patient along with cost and morbidity.

In our study an interesting fact was that out of 11 patients who had 3 or more positive lymph nodes, 10 were of luminal A type and only 1 was of luminal B type. Although triple-negative breast cancer patients and Her2-enriched type are considered to be more aggressive, this could be due to the small number of triple-negative breast cancer 
patients in the study group. Further studies need to be conducted to confirm these findings in case of axillary metastasis.

\section{Conclusion}

Intraoperative FS can be safely omitted in early breast cancer patients undergoing upfront breast conservation cancer surgery. ALND can be performed as a second operation if required post-chemotherapy as the percentage of such patients is very small. Although difficult to define a specific group, FS can be performed in selected group of patients with clinically suspicious looking nodes, large primary tumour size and poor tumour biology.

\section{Conflict of Interest}

The authors declare that they have no conflict of interest.

\section{References}

1. Bland CS. The Halsted mastectomy: Present illness and past history. West J Med 1981;134:549-55.

2. Fisher B, Wolmark N, Redmond C, et al. Findings from NSABP protocol no. B-04: Comparison of radical mastectomy with alternative treatments. II. The clinical and biologic significance of medial-central breast cancers. Cancer 1981;48:1863-72.

3. Fisher B, Bauer M, Margolese R, et al. Five-year results of a randomized clinical trial comparing total mastectomy and segmental mastectomy with or without radiation in the treatment of breast cancer. $\mathrm{N}$ Engl J Med 1985;312:665-73.

4. Giuliano AE, Kirgan DM, Guenther JM, et al. Lymphatic mapping and sentinel lymphadenectomy for breast cancer. Ann Surg 1994;220:391-8.

5. Krag D, Weaver D, Ashikaga T, et al. The sentinel node in breast cancer a multicenter validation study. $\mathrm{N}$ Engl J Med 1998;339:941-6.

6. Veronesi U, Paganelli G, Viale G, et al. A randomized comparison of sentinel-node biopsy with routine axillary dissection in breast cancer. N Engl J Med 2003;349:546-53.

7. Krag DN, Anderson SJ, Julian TB, et al. Sentinel-lymphnode resection compared with conventional axillarylymph-node dissection in clinically node-negative patients with breast cancer: Overall survival findings from the NSABP B-32 randomised phase 3 trial. Lancet Oncol 2010;11:927-33.

8. Fortunato L, Amini M, Farina M, et al. Intraoperative examination of sentinel nodes in breast cancer: Is the glass half full or half empty? Ann Surg Oncol 2004;11:1005-10.

9. Weiser MR, Montgomery LL, Susnik B, et al. Is routine intraoperative frozen-section examination of sentinel lymph nodes in breast cancer worthwhile? Ann Surg Oncol 2000;7:651-5.

10. van de Vrande S, Meijer J, Rijnders A, et al. The value of intraoperative frozen section examination of sentinel lymph nodes in breast cancer. Eur J Surg Oncol 2009;35:276-80.

11. Arora N, Martins D, Huston TL, et al. Sentinel node positivity rates with and without frozen section for breast cancer. Ann Surg Oncol 2008;15:256-61.

12. Lyman GH, Giuliano AE, Somerfield MR, et al. American society of clinical oncology guideline recommendations for sentinel lymph node biopsy in early-stage breast cancer. J Clin Oncol 2005;23:7703-20.

13. Giuliano AE, McCall L, Beitsch P, et al. Locoregional recurrence after sentinel lymph node dissection with or without axillary dissection in patients with sentinel lymph node metastases: The American college of surgeons oncology group Z0011 randomized trial. Ann Surg 2010;252:426-32.

14. Bishop JA, Sun J, Ajkay N, et al. Decline in frozen section diagnosis for axillary sentinel lymph nodes as a result of the American college of surgeons oncology group Z0011 trial. Arch Pathol Lab Med 2016;140:830-5.

15. Gentilini O, Veronesi U. Abandoning sentinel lymph node biopsy in early breast cancer? A new trial in progress at the European institute of oncology of Milan (SOUND: Sentinel node vs observation after axillary UltraSouND). Breast 2012;21:678-81.

16. Rónká R, Smitten KV, Sintonen H, et al. The impact of sentinel node biopsy and axillary staging strategy on hospital costs. Ann Oncol 2004;15:88-94.

17. Lim J, Govindarajulu S, Sahu A, et al. Multiple step-section frozen section sentinel lymph node biopsy a review of 717 patients. Breast 2013;22:639-42.

18. Poling JS, Tsangaris TN, Argani P, et al. Frozen section evaluation of breast carcinoma sentinel lymph nodes: A retrospective review of 1,940 cases. Breast Cancer Res Treat 2014;148:355-61.

19. Russo L, Betancourt L, Romero G, et al. Frozen section evaluation of sentinel lymph nodes in breast carcinoma: Aretrospective analysis. Ecancermedicalscience 2017;11:774.

\section{Authorship Contributions}

Concept and design: RNK, AA, SM, AIK, MAP, AL; Data Collection and interpretation: RNK, AA, SM; Literature review and writing: RNK, AA; Manuscript approval: RNK, AA, SM, AIK, MAP, AL 